\title{
Beschäftigungsperspektiven für Langzeitarbeitslose - ABM, Arbeitsgelegenheiten und öffentlich finanzierte Beschäftigung
}

Die Umsetzung der Hartz-Gesetze war mit massiven Veränderungen in der arbeitsmarktpolitischen Beschäftigungsförderung verbunden. Traditionelle Arbeitsbeschaffungsmaßnahmen wurden stark reduziert, Arbeitsgelegenheiten mit Mehraufwandsentschädigung in breitem Umfang eingesetzt. Inzwischen wird eine Ausweitung öffentlich finanzierter Beschäftigung für einen eng definierten Personenkreis in neuen Förderformen vorgeschlagen. Dieser Prozess ist begleitet von kontroversen Diskussionen über Förderphilosophie, Art und Umfang des Instrumenteneinsatzes sowie unterschiedlichen Interpretationen der empirischen Befunde über die Wirkungen des bisherigen Maßnahmeeinsatzes.

\section{Einleitung}

Maßnahmen zur Beschäftigungsförderung gehören zu den klassischen Instrumenten der Arbeitsmarktpolitik. Beschäftigung fördernde Maßnahmen ${ }^{1}$ sollen - wie generell alle Instrumente der aktiven Arbeitsmarktpolitik - sowohl auf unterschiedliche Arbeitsmarktgegebenheiten als auch auf die individuellen Voraussetzungen und Bedürfnisse der Arbeit suchenden Menschen reagieren. Auf Arbeitsmärkten mit erheblichem Arbeitsplatzdefizit können sie nachfragesteigernd wirken und so zur Entlastung des Arbeitsmarktes beitragen. Ebenso können sie die Eingliederung besonders förderungsbedürftiger Personen unterstützen.

Über den arbeitsmarkt- und beschäftigungspolitischen Nutzen öffentlich geförderter Beschäftigung wird jedoch heftig gestritten. Selbst die Befürworter haben in Bezug auf Zielgruppen, Einsatzfelder, Ausgestaltung und Perspektiven sehr unterschiedliche Auffassungen. Während das Bundesministerium für Arbeit und Soziales (BMAS) im Ergebnis der ersten Etappe der Evaluation der Hartz-Gesetze zu einer eher kritischen Einschätzung von Arbeitsbeschaffungsmaßnahmen (ABM) kam (BMAS 2006, S. V), schlägt die Koalitionsarbeitsgruppe Arbeitsmarkt inzwischen eine Ausweitung öffentlich finanzierter Beschäftigung in neuen Formen vor (BMAS 2007, S. 16ff.). Was auf den ersten Blick als Widerspruch erscheinen mag, zeigt tatsächlich einen Wandel in der Förderphilosophie und einen sich fortsetzenden Pro- zess der Segmentierung arbeitsmarktpolitischer Angebote, der bereits in der Teilung in zwei Rechtskreise (SGB III und SGB II) sowie in der Kundendifferenzierung bei der Bundesagentur für Arbeit (BA) sichtbar wurde.

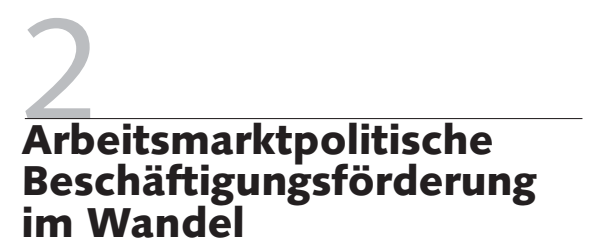

\subsection{AUSWEITUNG DER NACHFRAGE- STÄRKENDEN INSTRUMENTE...}

Die Ziele und die Ausgestaltung der arbeitsmarktpolitischen Beschäftigungsförderung veränderten sich im Zeitverlauf. Ausschlaggebend dafür waren sowohl Entwicklungen auf dem Arbeitsmarkt als auch veränderte politische Einschätzungen darüber, wie Arbeitsmarktpolitik unter gegebenen Bedingungen agieren sollte.

Zur Entstehungszeit des Arbeitsförderungsgesetzes (AFG) 1969 ging es vor allem darum, mit Beschäftigungsmaßnahmen das Tal einer Rezession zu überbrücken. In den 1970er und 1980er Jahren sind ABM aufgrund der steigenden Arbeitslosigkeit quantitativ ausgeweitet und durch Sonderprogramme zusätzlich gefördert worden. Parallel dazu entwickelte sich ab Anfang der 1980er Jahre eine breite kommunale Beschäftigungsförderung als Reaktion auf die wachsende Sozialhilfebedürftigkeit wegen Arbeitslosigkeit. Im Zusammenhang mit den Anpassungsprozessen auf dem ostdeutschen Arbeitsmarkt wurden ABM seit
1990 in quantitativ und qualitativ neuer Dimension eingesetzt, um sowohl den Sturz in die Marktwirtschaft abzumildern (sogenannte Fallschirmfunktion) als auch den damit verbundenen wirtschaftlichen Strukturwandel zu flankieren (vgl. u. a. Seifert/Wagner 1992; Knuth 1996). Zu dieser Zeit wurde in der arbeitsmarktpolitischen Diskussion die Frage aufgeworfen, ob die traditionelle Arbeitsmarktpolitik unter den Bedingungen eines hohen allgemeinen Beschäftigungsdefizits nicht ergänzt werden sollte um komplexere Zielstellungen und Funktionen, neue Instrumente und Finanzierungsmechanismen. Vorstellungen über einen umfassenden „Zweiten Arbeitsmarkt" wurden sehr kontrovers diskutiert (Wagner 1995; Trube 1997). Schon damals ging es nicht nur um die Ausweitung geförderter sozialversicherungspflichtiger Beschäftigung, sondern ebenso um die Förderung von Sozialrechtsverhältnissen in Form der Beschäftigung arbeitsloser Sozialhilfeempfänger nach $₫ 19$ Bundes-

\footnotetext{
Darunter werden im Folgenden solche Maßnahmen verstanden, durch die zusätzliche Arbeitsplätze geschaffen werden. Nicht gemeint sind Lohnkostenzuschüsse zur Unterstützung der Eingliederung arbeitsloser Personen in Beschäftigung.
}

Alexandra Wagner, Dr., Geschäftsführerin des FIA (Forschungsteam Internationaler Arbeitsmarkt). Arbeitsschwerpunkte: Arbeitsmarktreformen und Arbeitsmarktpolitik, Frauenerwerbstätigkeit, Arbeitszeitgestaltung im Betrieb, Führungskräfte und Firmenkultur. e-mail:wagner@fia-institut.de 
sozialhilfegesetz (BSHG)2 oder in ab 1994 möglichen freiwilligen Gemeinschaftsarbeiten für Arbeitslosenhilfeempfänger. ${ }^{3}$

Im Zuge der Diskussion über einen breiteren Einsatz von Beschäftigungsfördermaßnahmen kam es zur Modifizierung bisheriger und zur Einführung neuer Instrumente. Neben das traditionelle Instrument der Beschäftigungsförderung, die ABM, traten die 1993 eingeführten pauschalierten Lohnkostenzuschüsse zur Arbeitsförderung ( $\$ 249 \mathrm{~h} \mathrm{AFG)}$ ) als ein neues Instrument (vgl. u. a. Knuth/Wagner 1995). Dieses Instrument bot die Chance, die vorrangig personenbezogene Zielgruppenförderung mit $\mathrm{ABM}$ durch eine präventive und eher sachzielorientierte beschäftigungswirksame Projektförderung mit längerfristiger Perspektive zu ergänzen. Mit dem $\$ 249$ h war eine Förderung nur in definierten Einsatzfeldern zur Infrastrukturförderung möglich, und die Projekte hatten eine im Vergleich zu ABM längere Förderdauer. Außerdem trug dieses Instrument durch die Umwandlung passiver Leistungen in Mittel zur Arbeitsförderung auf sehr anschauliche Weise der Forderung Rechnung, Arbeit statt Arbeitslosigkeit zu finanzieren. Parallel wurden mit den „Gesellschaften zur Arbeitsförderung, Beschäftigung und Strukturentwicklung" (ABS-Gesellschaften) auch qualitativ neue Träger der Arbeitsmarktpolitik etabliert (Knuth 1996).

Die Beschäftigungsförderung erlangte damit eine andere Logik: Sie war nicht nur Hilfe für Personen mit Vermittlungshemmnissen, sondern erfüllte gleichzeitig wichtige wirtschafts-, regional- und strukturpolitische Funktionen; sie war auch im Bereich privater Wirtschaftsunternehmen einsetzbar, orientierte auf die Schaffung von Ersatzarbeitsplätzen für die aktuell auf andere Weise nicht in Arbeit integrierbaren Personen und ermöglichte projektbezogene und längere Förderdauern. Diese Förderlogik wurde später durch den $\$ 242$ s AFG auch für die alten Bundesländer übernommen und mündete schließlich in das Instrument der Strukturanpassungsmaßnahmen (SAM), die mit Hartz III ab Januar 2004 als eigenständiges Instrument aufgehoben und mit den ABM zusammengeführt wurden.

\section{2 ...UND UMSTEUERUNG AUF "AKTIVIERENDE" ARBEITSMARKTPOLITIK}

Förderphilosophie und Instrumenteneinsatz unterlagen auch in der jüngsten Ver- gangenheit einem grundlegenden Wandel. Die „Hartz-Gesetze“ zielten darauf ab, den Bestand an gemeldeter Arbeitslosigkeit unabhängig vom makroökonomischen Umfeld durch Beeinflussung des individuellen Arbeitsmarktverhaltens Arbeitsuchender zu verringern. Mit dem Übergang von einer aktiven zu einer „aktivierenden“ Arbeitsmarktpolitik verband sich die Idee, den Bestand an gemeldeter Arbeitslosigkeit vorrangig durch eine schnellere Besetzung offener Stellen aufgrund besserer Vermittlung und erhöhter Konzessionsbereitschaft der Arbeitsuchenden zu erreichen. Dieser Politikwechsel, der bereits 1998 mit der Neufassung des Zielkatalogs für Arbeitsförderung im SGB III begann und 2002 mit dem Job-AQTIV-Gesetz weiter vorangetrieben wurde, ist mit der Fürsorgelogik des SGB II endgültig vollzogen. Er geht einher mit einer skeptischen Kosten-NutzenEinschätzung klassischer Instrumente der Arbeitsförderung, insbesondere der direkt Beschäftigung schaffenden Maßnahmen (Bartelheimer/Wagner 2005).

Dies zeigt sich nicht nur in einer zahlenmäßigen Rückführung von $\mathrm{ABM}$ (Abschnitt 4), sondern auch in einer sukzessiven Verschlechterung der Förderkonditionen, die sich in einer Vielzahl von Rechtsänderungen niederschlugen. Hervorzuheben sind die Änderung der Zielbestimmung von ABM (Abschnitt 4.1), die Ablösung des bisherigen Lohnkostenzuschusses in Höhe eines bestimmten Prozentsatzes des jeweiligen berücksichtigungsfähigen Entgelts durch einen (qualifikationsabhängigen) pauschalen $\mathrm{Zu}$ schuss, der Wegfall der Orientierung an tariflichen oder ortsüblichen Arbeitsentgelten und die Streichung des Arbeitslosenversicherungsbeitrages. Bis 2003 war es ein gewünschter Effekt, dass über eine tariflich bzw. ortsüblich entlohnte ABMBeschäftigung (neue) Ansprüche auf Leistungen der Arbeitslosenversicherung erworben werden konnten. Im Zusammenwirken von mehrfach verschärften Zumutbarkeitsregelungen und verschlechterten Förderkonditionen hat das in $\$ 1$ SGB III formulierte Ziel, unterwertiger Beschäftigung entgegen zu wirken, in der praktischen Umsetzung der Beschäftigungsförderung keine wahrnehmbare Wirkung. Das SGB II kennt ohnehin keinerlei Zumutbarkeitsgrenzen.

\section{Zwei Formen öffentlich finanzierter Beschäftigung}

Ein großer Teil der arbeitsmarktpolitischen Beschäftigungsförderung ist heute komplett öffentlich finanziert, d. h. die Lohnkosten für geförderte Personen werden vollständig aus Mitteln der Arbeitsmarktpolitik gedeckt. ${ }^{4}$ Gegenüber anderen Formen öffentlich finanzierter Beschäftigung besteht die Spezifik der arbeitsmarktpolitischen Beschäftigungsförderung darin, dass die Stellenbesetzung nach arbeitsmarktund sozialpolitischen Kriterien erfolgt. Gemeinsam sind dem öffentlichen Dienst und der arbeitmarktpolitisch geförderten Beschäftigung, dass es sich um Tätigkeiten handelt, die im gesellschaftlichen Interesse liegen. Eine Verbindung zwischen beiden Formen wird darüber hinaus über das Kriterium der "Zusätzlichkeit“ deutlich: Arbeitsmarktpolitik darf überwiegend nur dort Arbeitsplätze schaffen, wo Privatwirtschaft und öffentlicher Dienst Lücken lassen. „Zusätzlich“ in diesem Sinne können auch Tätigkeiten sein, die zwar von ihrer Bestimmung her durch den öffentlichen Dienst übernommen werden können oder sollten, jedoch - z. B. aufgrund der Haushaltslage - innerhalb einer bestimmten Frist real nicht geleistet werden. Jenseits der (nicht übertragbaren) Pflichtaufgaben des Öffentlichen Dienstes gibt es folglich ein breiteres Spektrum von freiwilligen öffentlichen Leistungen, die im gesellschaftlichen Interesse liegen.

Noch in den 1970er Jahren stand außer Frage, dass die Personalentwicklung im öffentlichen Dienst eine wichtige politische Stellschraube zur Milderung von Arbeitsmarktproblemen ist. In von der BA ange-

\footnotetext{
2 Dies konnten Arbeitsgelegenheiten mit Arbeitsentgelt sein, aber auch solche, bei denen die Hilfe zum Lebensunterhalt zuzüglich einer Entschädigung für Mehraufwendungen gewährt wurde.

3 Mit dem Beschäftigungsförderungsgesetz 1994 (BeschfG) wurde in das AFG der §134 Abs. 3b neu eingefügt, wonach der Anspruch auf Arbeitslosenhilfe weiterhin besteht, wenn Arbeitslose gemeinnützige und zusätzliche Arbeit im Sinne des $\S 19$ Abs. 3 des BSHG verrichten.

4 Es gibt kaum noch Träger, die in der Lage sind, die pauschalen Zuschüsse für $A B M$ so aufzustocken, dass das Niveau eines tariflichen Entgelts erreicht wird.
} 
stellten „Überlegungen II zu einer vorausschauenden Arbeitsmarktpolitik" heißt es: „Wenn offenkundig einerseits bestimmte wichtige Bedürfnisse nur durch verstärkte Beteiligung der öffentlichen Haushalte an der Finanzierung entsprechender Dienstleistungen und Infrastruktureinrichtungen befriedigt werden können, wenn andererseits die zur Erbringung dieser Leistungen bzw. zum Bau dieser Infrastruktur benötigten Arbeitskräfte ohne Beschäftigung sind und als Arbeitslose nicht viel geringere direkte und indirekte öffentliche Kosten verursachen, als ihre sinnvolle Beschäftigung mit Hilfe verstärkter öffentlicher Ausgaben erfordern würde, ist weniger nach den Auswirkungen auf die Staatsquote zu fragen. Es wäre vielmehr darüber nachzudenken, wie die Mittel am vernünftigsten, also mit den wenigsten negativen Nebeneffekten aufgebracht werden sollen. (...) Dem öffentlichen Dienst wird damit in gewisser Hinsicht eine Entlastungsaufgabe für den Arbeitsmarkt zuerkannt" (Bundesanstalt für Arbeit 1978). An diesen Überlegungen fällt auf, dass sie an die Bedingung geknüpft waren, dass die Kosten zur Finanzierung der Arbeitslosigkeit kaum geringer sind als die Kosten der öffentlichen Beschäftigung.

Die heutige Situation ist völlig anders: Der öffentliche Dienst stützt das Beschäftigungsniveau nicht mehr. Im Gegenteil: Er trägt durch kontinuierliche Personalreduzierungen mit zur angespannten Arbeitsmarktsituation bei. Hinzu kommt, dass Absenkungen im Leistungsniveau des Arbeitslosengeldes, Übertritte von Arbeitslosen in das Grundsicherungssystem und Senkungen der Standards bei öffentlich geförderter Beschäftigung das oben genannte Argument der weitgehenden Kostenneutralität öffentlich finanzierter Beschäftigung schwächen: Gerade weil die gleichen Tätigkeiten heute durch öffentlich geförderte Beschäftigung weitaus billiger ausgeführt werden können, wird der Ersatz regulärer Beschäftigung durch ABM oder Arbeitsgelegenheiten (AGH) finanziell attraktiv und umgekehrt ihre Überführung in ungeförderte öffentlich finanzierte Beschäftigung erschwert. Unter diesen Bedingungen besteht das Risiko, dass der Einsatz arbeitsmarktpolitischer Instrumente den Personalabbau im öffentlichen Dienst sogar fördert. Die Gestaltung der Schnittstelle zwischen öffentlich finanzierter Beschäftigung in regulärer und arbeitsmarktpolitisch geförderter Form ist folglich von ent-

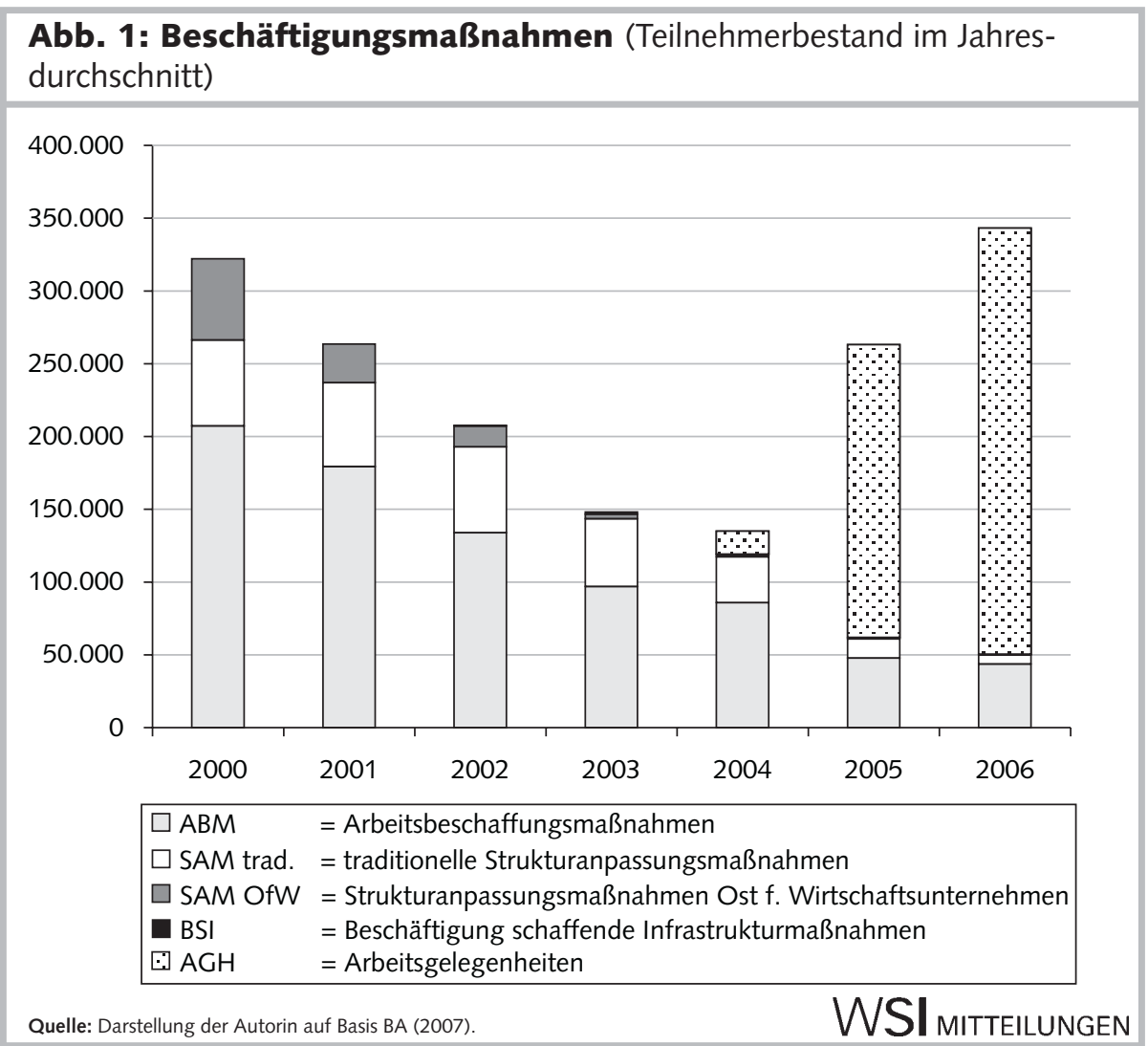

scheidender Bedeutung für deren beschäftigungspolitische Wirksamkeit.

\section{1 \\ Aktuelle Entwicklungen arbeitsmarktpolitischer Beschäftigungsförderung}

Infolge der Umsetzung der Hartz-Gesetze und der neuen Geschäftspolitik der BA hat die arbeitsmarktpolitische Beschäftigungsförderung einen Strukturwandel erfahren: Der jahresdurchschnittliche Bestand bei ABM sank von 2000 bis 2006 um ca. $80 \%$ auf 43.700 Teilnehmerinnen und Teilnehmer. Rückläufig war außerdem die durchschnittliche Laufzeit der Maßnahmen. Von den ABM im Jahr 2006 entfielen über $80 \%$ auf das SGB II, der ABM-Teilnehmerbestand im SGB III lag bei jahresdurchschnittlich nur noch 8.300 Personen. Demgegenüber hat die Zahl der in AGH-Geförderten seit 2004 so deutlich zugenommen, dass der Rückgang bei ABM und SAM rein zahlenmäßig sogar überkompensiert werden konnte (Abbildung 1). Unter den AGH bilden solche mit Mehraufwandsentschädigung (sogenannte Zusatz- oder Ein-Euro-Jobs) die überwiegende Mehrheit (94\% des jahresdurchschnittlichen Teilnehmerbestands in 2006), während AGH in sozialversicherungspflichtiger Form bislang nur eine untergeordnete Rolle spielen (jahresdurchschnittlich knapp 17.000 Teilnehmer in 2006).

Wollte man eine Gesamtbilanz ziehen, müssten auch die bis 2004 über das BSHG geförderten Maßnahmen einbezogen werden, für die es jedoch keine verlässliche Statistik gibt. Nach Angaben des Deutschen Städtetages (Fuchs/Troost 2003) wurden in den Jahren 2000 bis 2002 bundesweit insgesamt jeweils knapp 400.000 Personen gefördert.

\subsection{ZUR WIRKSAMKEIT VON ABM}

Die Wissenschaftler und Wissenschaftlerinnen, die für die Wirkungsforschung des BMAS zu den ersten drei Hartz-Gesetzen (BMAS 2006) das Instrument ABM evaluierten, haben auf Basis der gesetzlichen Regelungen fünf Ziele identifiziert, die mit der Durchführung von Beschäftigung schaffenden Maßnahmen verfolgt werden sollten: die Durchführung zusätzlicher, im öffentlichen Interesse liegender Arbeiten (Strukturwirksamkeit) mit besonders förderungsbedürftigen Personen (Zielgruppeninklusion), die berufliche und soziale 
Stabilisierung der Teilnehmenden mittels Einbeziehung in temporär geförderte Beschäftigungsmaßnahmen (Marktersatz), um damit den bei längerer Arbeitslosigkeit einsetzenden Dequalifizierungs- und Demotivierungstendenzen entgegenzuwirken (Erhalt bzw. Wiedererlangung der Beschäftigungsfähigkeit und Qualifizierung) und eine Verbesserung der Eingliederungsaussichten zu erreichen (Arbeitsmarktintegration) (Compass et al. 2006, S. 22). Dabei ist offensichtlich, dass mit einer konkreten Maßnahme kaum alle fünf Ziele gleichzeitig erreichbar sind. Es ist vielmehr davon auszugehen, dass das Instrument ABM vielfältig einsetzbar ist - für jeweils eines oder mehrere der genannten Ziele.

Mit Hartz III wurden ABM nicht nur neu geregelt, sondern auch mit einer neuen Zielbestimmung versehen. So heißt es in dem 2003 verabschiedeten Eckpunktepapier der Koalitionsarbeitsgruppe zur Gesetzesbegründung: „Arbeitsbeschaffungsmaßnahmen sollen insbesondere bei hoher Arbeitslosigkeit entsprechend den Problemschwerpunkten der regionalen und beruflichen Teilarbeitsmärkte gefördert werden. Arbeitslosigkeit soll hierdurch abgebaut und Arbeitslosen zur Erhaltung und Wiedererlangung ihrer Beschäftigungsfähigkeit zumindest vorübergehend eine Beschäftigung ermöglicht werden. Auf die ausdrückliche Umsetzung des Ziels Verbesserung der Eingliederungsaussichten der Arbeitnehmer, das in der Vergangenheit wegen der schlechten Arbeitsmarktlage kaum erreichbar war, kommt es bei diesem Instrument künftig nicht mehr an" (Koalitionsarbeitsgruppe 2003, S. 17). Diese Formulierung fand fast wörtlich Eingang in $\$ 260$ Abs. 1 SGB III. Damit sind „Ersatzbeschäftigung" und Erhalt bzw. Wiedererlangung der Beschäftigungsfähigkeit derzeit die vorgegebenen Ziele beim Einsatz von $\mathrm{ABM}$.

Insofern überrascht es, wenn das BMAS in seinem Abschlussbericht zur Evaluierung von Hartz I bis III (BMAS 2006, S. V) aufgrund der „negativen Integra-

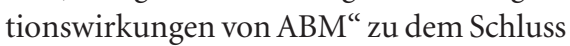
kommt, diese hätten sich ,als nicht erfolgreich erwiesen", und dies obwohl der Evaluationsbericht zu ABM (Compass et al. 2006) deren Wirkung sehr differenziert darstellt und bewertet:

- Die Untersuchung des Erhalts bzw. der Wiedererlangung von Beschäftigungsfähigkeit durch $\mathrm{ABM}$ ergab einen positiven Be- fund, insbesondere in Bezug auf die berufsfachlichen und gesundheitlichen Merkmale von Beschäftigungsfähigkeit. Bei der psychosozialen Dimension von Beschäftigungsfähigkeit ergab sich zwar eine Verbesserung während der $\mathrm{ABM}$, aber eine deutliche Verschlechterung nach Abschluss der Maßnahme.

- Im Hinblick auf die Marktersatzfunktion musste konstatiert werden, dass diese aufgrund der Geschäftspolitik der BA lediglich in einem quantitativ sinkenden Umfang realisiert werden konnte.

- In Bezug auf die (nach SGB III nicht mehr prioritären) Integrationswirkungen wurde festgestellt, dass insbesondere in Regionen mit guter Arbeitsmarktlage mit $\mathrm{ABM}$ für besonders förderungsbedürftige Personen positive Nettointegrationswirkungen erzielt werden konnten (Zielgruppeninklusion).

- Exemplarisch konnte darüber hinaus die Strukturwirksamkeit konkreter Maßnahmen belegt werden.

ABM ist vor dem Hintergrund der Ergebnisse der Wirkungsforschung keineswegs ein grundsätzlich erfolgloses Instrument. Der arbeitsmarktpolitische Erfolg von $A B M$ lässt sich allerdings nur im Kontext der konkreten Maßnahme beurteilen - je nachdem, ob sie z. B. in Regionen mit hohem Arbeitsplatzdefizit eher die Marktersatzfunktion erfüllen oder bei besserer Arbeitsmarktlage der konkreten Förderung von Zielgruppen dienen. Den Evaluatoren und Evaluatorinnen ist zuzustimmen: Geht es um den gesellschaftlichen Nutzen von $\mathrm{ABM}$, sind darüber hinaus weitere Kriterien der Bewertung heranzuziehen. Insbesondere ist mit zu bedenken, welche sozialen, gesundheitlichen und politischen Konsequenzen ein Verzicht auf ABM hätte. Eine Gesamtbewertung muss folglich über die rein arbeitsmarktbezogene Betrachtung hinausgehen und dürfte so die differenzierte, aber insgesamt positive Einschätzung von $A B M$ durch die Evaluation eher noch stärken.

\subsection{PROBLEMATISCHE WIRKUNGEN DER AGH}

Das SGB II fordert die Träger der Leistungen zur Grundsicherung für Arbeitsuchende in $\$ 16$ Abs. 3 explizit auf, unter bestimmten Bedingungen öffentlich geförderte Beschäftigung zu schaffen: „Für erwerbsfähige Hilfebedürftige, die keine
Arbeit finden können, sollen Arbeitsgelegenheiten geschaffen werden. " $\mathrm{Zu}$ den explizit im SGB II benannten Instrumenten zählen sowohl solche zur Schaffung von sozialversicherungspflichtiger Beschäftigung (ABM, AGH mit Entgelt) als auch Arbeitsgelegenheiten mit Mehraufwandsentschädigung (Zusatzjobs), die ebenfalls eine Form öffentlich geförderter Beschäftigung, allerdings in einem Sozialrechtsverhältnis, darstellen. Gemäß $\$ 16$ Abs. 1 in Verbindung mit Abs. 3 sind AGH mit Mehraufwandsentschädigung nachrangig gegenüber allen anderen Förderinstrumenten, insbesondere jenen, die inhaltlich auf dem SGB III fußen (vgl. dazu auch BA 2004, S. 64). Sie werden daher zu Recht auch als arbeitsmarktpolitische ultima ratio bezeichnet.

Die oben dargestellte Entwicklung zeigt jedoch, dass die Zusatzjobs gegenwärtig zum dominierenden Instrument der arbeitsmarktpolitischen Beschäftigungsförderung geworden sind. Wesentliche Ursachen dafür liegen darin, dass für die Grundsicherungsträger die Förderung sozialversicherungspflichtiger Beschäftigung deutlich aufwändiger ist als die von $\mathrm{Zu}$ satzjobs ${ }^{5}$ und dass die Zusatzjobs für die Träger aufgrund der Teilnehmerpauschale finanziell attraktiv sind. Außerdem werden die Zusatzjobs häufig lediglich zum Test der Arbeitsbereitschaft eingesetzt und dienen damit nicht primär arbeitsmarktpolitischen Zielen. Die Betroffenen sind zur Annahme des Angebots verpflichtet - selbst dann, wenn die Maßnahmen nicht passgenau sind. Bei Ablehnung drohen Sanktionen in Form von Leistungskürzungen.

Die in Zusatzjobs „Beschäftigten“ werden statistisch als Erwerbstätige erfasst, obwohl sie sich in keinem regulären Beschäftigungsverhältnis befinden und kein Arbeitsentgelt erhalten. Sie bleiben in vollem Umfang auf die SGB-Leistungen (Arbeitslosengeld II und Kosten der Unterkunft) angewiesen, und für sie ist das gesamte Individual- und Kollektivarbeitsrecht grundsätzlich nicht anwendbar. Trotz dieser wenig komfortablen Beschäftigungsbedingungen bemühen sich aufgrund fehlender

\footnotetext{
Während für die Zusatzjobs aufgrund der Weiterzahlung der Leistungen nur geringe Mehrkosten (Mehraufwandsentschädigung und Trägerpauschale) anfallen, müssen AGH mit Entgelt vollständig aus dem Eingliederungstitel finanziert werden.
} 
Alternativen auch solche Hilfebeziehenden um diese Jobs, die eigentlich andere Unterstützungsleistungen benötigen würden. Zusatzjobs sind auch deshalb attraktiv, weil die Mehraufwandsentschädigung eine geringfügige Verbesserung der finanziellen Situation verspricht und die Einbindung in ein neues soziales Umfeld positiv bewertet wird. Insofern besteht die Gefahr eines "Creamings", also von Fehlsteuerungen, mit den entsprechenden Benachteiligungen für die eigentliche Zielgruppe der besonders Förderungsbedürftigen. Vorliegende wissenschaftliche Analysen bestätigen, dass die Zusatzjobs bislang nicht gezielt zur Förderung von besonders schwer vermittelbaren Arbeitslosen eingesetzt werden $^{6}$ (Wolff/Hohmeyer 2006).

Arbeitsmarktpolitisch bedenklich ist darüber hinaus, dass Zusatzjobs selbst für prinzipiell geeignete Personen kaum Brücken in den ungeförderten Arbeitsmarkt bieten (Kettner/Rebien 2007). In Ostdeutschland ist davon auszugehen, dass AGHs sozialversicherungspflichtige Beschäftigung verdrängen (Hohendanner 2007). Indizien weisen darauf hin, dass bundesweit etwa jeder zweite Betrieb, der das Instrument nutzt, zumindest einen Teil seiner beschäftigten Zusatzjobber nicht im Sinne des Gesetzgebers einsetzt (Kettner/ Rebien 2007), sondern diese z. B. für Krankheitsvertretungen, Überstundenabbau der regulären Belegschaft oder gar zum direkten Personalabbau (direkte Substitution von Arbeitsplätzen) nutzt. Häufig würden Arbeiten der regulär Beschäftigten ,schleichend" auf Zusatzjobber übertragen, was längerfristig ebenfalls zum Personalabbau führen kann, zumal die Tätigkeiten nach Auslaufen der Zusatzjobs durch neue $\mathrm{Zu}$ satzjobber übernommen werden (ebd.). Insofern ist der Hinweis sehr plausibel, dass sich die Wirtschafts- und Arbeitsmarktpolitik derzeit zu wenig um die gesamtwirtschaftlichen Risiken der Ein-Euro-Jobs kümmert. Damit verbunden sind nachhaltig negative Konsequenzen: Ohne ein verantwortliches Wirken der Akteure können sich AGHs zu einem Instrument entwickeln, ,das dem Arbeitsmarkt insgesamt mehr schadet als nützt" (ebd., S. 62). Wenn die Zusatzjobs die ihnen zugedachte Funktion als persönliches Hilfeangebot zur Eingliederung in Arbeit und Gesellschaft erfüllen sollen, muss ihr Einsatz quantitativ beschränkt bleiben, um die Qualität zu erhalten und Mitnahmeeffekte zu verhindern (Spindler 2005).

\section{Die aktuelle politische Diskussion}

Aktuell erfährt die Diskussion über die arbeitsmarktpolitische Beschäftigungsförderung einen neuen Schub. Ausgangspunkt vielfältiger Vorschläge ${ }^{7}$ zu ihrer Ausweitung und qualitativen Veränderung 8 sind das weiter bestehende Arbeitsplatzdefizit und die damit verbundene Verfestigung von Langzeitarbeitslosigkeit, während gleichzeitig im sozialen und ökologischen Bereich Arbeiten unerledigt bleiben und öffentlich finanzierte Beschäftigung in sozialversicherungspflichtiger Form letztlich nur mit geringen Mehrkosten im Vergleich zur „passiven Alimentierung“ der Arbeitslosen verbunden ist. Hinzu kommt eine kritische Sicht auf Einsatz und Wirkungen der Zusatzjobs.

Das Spektrum der inhaltlichen Vorschläge ist breit und reicht von einem umfangreichen Einsatz öffentlich finanzierter Ersatzbeschäftigung zu tariflichen Bedingungen und Mindestentgelten von monatlich $1.400 €$ bei Sozialversicherungspflicht einschließlich dem Beitrag zur BA und ausschließlich freiwilligem Zugang (Linksfraktion) bis zur derzeit im Modellversuch praktizierten verpflichtenden „Bürgerarbeit" für Entgelte, die das Arbeitslosengeld II kaum übersteigen und (auch) dem Ziel dienen, sogenannte „unechte“ Arbeitslose aus der Arbeitsmarktstatistik zu entfernen (Sachsen-Anhalt). Die meisten Vorschläge beziehen sich jedoch übereinstimmend auf ein bestimmtes Segment, nämlich die Integration langzeitarbeitsloser bzw. sogenannter „arbeitsmarktferner"9 Personen.

\subsection{VORSCHLAG DER KOALITIONSARBEITSGRUPPE}

Der Vorschlag der Koalitionsarbeitsgruppe Arbeitsmarkt vom April diesen Jahres (BMAS 2007; Brandner 2007) stellt in gewisser Weise einen vorläufigen Endpunkt der Regierungsdiskussion dar. Ausgehend von der Diagnose, dass ein Teil der SGB IILeistungsbeziehenden aufgrund besonderer Vermittlungshemmnisse voraussichtlich dauerhaft auf Fürsorgeleistungen angewiesen bleiben wird und die Integration dieser Personen in reguläre Beschäftigung auch bei verbesserter Konjunktur mit tra- ditionellen Mitteln der Arbeitsmarktpolitik nicht möglich ist, wird vorgeschlagen, für einen bestimmten Personenkreis unbefristete sozialversicherungspflichtige Beschäftigungsmöglichkeiten (allerdings ohne Beitrag zur BA) zu schaffen. Während in der vorangegangenen Diskussion noch von 400.000 zu fördernden Personen die Rede war (vgl. u. a. Alt 2007), orientiert die Koalitionsarbeitsgruppe nun auf 100.000 entsprechende Arbeitsplätze, die ab Januar 2008 bis zum Ende der laufenden Legislaturperiode eingerichtet werden sollen. Diese Beschäftigung soll tarif- oder ortsüblich entlohnt werden. Als Einsatzfelder werden genannt: Tätigkeiten im gewerblichen und sozialen Bereich bei Unternehmen des ersten Arbeitsmarktes, sozialen Betrieben und Integrationsunternehmen. Erforderlich sei es, dass die Betriebe zumindest eine teilweise Kostendeckung durch eigene Einnahmen erreichen.

Als Zielgruppe für diese neuen Fördermöglichkeiten werden solche Menschen angesehen, „die nach realistischer Erwartung innerhalb der nächsten 24 Monate keine Chance haben, einen Platz auf dem regulären Arbeitsmarkt zu finden“. Dazu gehörten Arbeitslose, die länger als ein Jahr arbeitslos sind, bei denen der Einsatz arbeitsmarktpolitischer Hilfen bisher erfolglos war und die besonders schwere Vermittlungshemmnisse aufweisen. Bei Beschäftigung dieser Personen sollen Arbeitgeber einen Zuschuss in Form eines Nachteilsausgleichs erhalten. Voraussetzungen sind eine Vollzeittätigkeit, mindestens jedoch eine Arbeitszeit von $50 \%$ der regulären Arbeitszeit und eine Entlohnung

6 Dies gilt übrigens auch - wie Hohmeyer et al (2006) zeigen konnten - für die AGH in der Entgeltvariante.

7 U. a. von der Linksfraktion im Deutschen Bundes tag (BT-Drs. 16/2504), von der Fraktion von Bündnis 90/Die Grünen (BT-Drs. 16/2652), dem DGB (2006), von der Bundesagentur für Arbeit, von der bag arbeit e. V., der BAGFW, dem Deutschen Verein, dem Paritätischen Wohlfahrtsverband (alle Vorschläge in: Deutscher Bundestag. Ausschuss für Arbeit und Soziales (2007)).

8 Eine Ausnahme bildet die BDA, die eine öffentlich geförderte Beschäftigung entschieden ablehnt und sich dabei auf die im BMAS-Bericht zur HartzEvaluierung enthaltene negative Beurteilung der ABM beruft.

9 Dieser Begriff ist irreführend, da arbeitslose Personen sich per definitionem auf dem Arbeitsmarkt befinden. Gemeint ist, dass sie bereits seit Längerem keine Beschäftigung mehr hatten oder noch nie erwerbstätig waren. 
mindestens in Höhe der örtlich maßgeblichen untersten Tarifgruppe oder einer vergleichbaren ortsüblichen Entlohnung. Bei einer Vollzeittätigkeit darf die Entlohnung nicht niedriger sein als die Summe aus Regelsatz, Kosten der Unterkunft und Heizung und Erwerbstätigenfreibetrag, d. h. es soll die Hilfebedürftigkeit (einer alleinstehenden Person) überwunden werden. $\mathrm{Zu}$ sätzlich sollen Zuschüsse möglich sein, wenn die Arbeitgeber zum Aufbau von Beschäftigungsmöglichkeiten einen besonderen Aufwand nachweisen. Arbeitnehmer und Arbeitnehmerinnen sollen darüber hinaus durch Weiterbildungsmaßnahmen und psychosoziale Dienstleistungen unterstützt werden. Die Förderung soll ggf. auch dauerhaft möglich sein, wobei die Fördervoraussetzungen alle zwölf Monate zu überprüfen sind.

\subsection{SONDERLÖSUNGEN ZULASTEN EINES GESAMTKONZEPTS?}

Der Vorschlag der Arbeitsgruppe findet zwar breite Unterstützung, gleichwohl werden wichtige Fragen durchaus kontrovers diskutiert (ausführlich dazu Koch/Kupka 2007).

Politisch weitgehend einig ist man sich über den grundsätzlichen Handlungsbedarf. Gleichzeitig gibt es hohe Übereinstimmung, dass die bisherige auf Integration in den ungeförderten Arbeitsmarkt gerichtete Förderphilosophie zumindest ergänzt werden muss um eine längerfristige Orientierung auf Marktersatz, soziale Stabilisierung und Erschließung der vorhandenen Ressourcen der bzw. einer bestimmten Gruppe von Arbeitslosen, was sich in längerfristiger Förderung niederschlagen muss. Dies kann durchaus als partielle Korrektur der Arbeitsmarktpolitik der letzten Jahre interpretiert werden. Unstrittig ist auch, dass sowohl die Auswahl der Personen als auch die Bestimmung der Einsatzfelder vor Ort im Zusammenwirken der lokalen Akteure erfolgen müssen. Die Unterschiede in den Positionen liegen vor allem im Umfang der angestrebten Förderung, in der Definition von Zielgruppen, der Höhe der Entlohnung, dem Ein- oder Ausschluss der Arbeitslosenversicherungspflicht, der Freiwilligkeit des Zugangs u. ä. Diese Differenzen sind keineswegs nachrangig. Vielmehr widerspiegeln sie ein unterschiedliches Herangehen im Grundsatz. Insofern ist die Differenziertheit der Meinungen breiter, als es auf den ersten Blick scheinen mag.
Kontrovers diskutiert wird allerdings auch die Grundkontur der angestrebten (Neu-)Ausrichtung der Beschäftigungsförderung in der Arbeitsmarktpolitik, die zwar auf quantitativen Ausbau und längere Förderdauern zielt, dies jedoch nur einer kleinen Gruppe von Arbeitslosen zugestehen will. Statt der Konzentration auf ein bestimmtes Segment und der nicht begründbaren Unterteilung der Arbeitslosen „in Spreu und Weizen“ (Haberkorn 2006) sei vielmehr ein schlüssiges Gesamtkonzept nötig. Die vorhandenen Instrumentarien seien völlig ausreichend für differenzierte Eingliederungs- und Beschäftigungskonzepte der unterschiedlichsten Zielgruppen. Das Problem läge vielmehr darin, dass diese bislang nicht richtig genutzt worden seien. Für diejenigen, denen geholfen werden soll, sei es unter Umständen sogar kontraproduktiv, wenn ihnen ihre Integrationsfähigkeit tendenziell abgesprochen würde. Es könnte aber auch sein, dass Personen einmal für die dauerhafte geförderte Beschäftigung ausgewählt - keine Motivation mehr entwickeln, diese zugunsten einer regulären Beschäftigung zu verlassen. Schließlich wird auf den Widerspruch aufmerksam gemacht, der zwischen der Defizitbeschreibung der Zielgruppe und der für sie angedachten Aufgabe der Erschließung neuer Beschäftigungsfelder in sozialen Dienstleistungen besteht. In diesem Kontext wird die Befürchtung geäußert, dass Misserfolge zu einer Diskreditierung der Arbeitsförderung im Allgemeinen und der öffentlich geförderten Beschäftigung im Besonderen führen könnten (Schomburg 2007, S. 7).

\section{Ausblick}

Die aktuelle Diskussion zeigt den dringenden Bedarf an Beschäftigungsförderung in der Arbeitsmarktpolitik und verweist auf Defizite ihrer Umsetzung in der jüngsten Vergangenheit. Stärker als bislang wird auf die Mehrdimensionalität der Ziele und die positiven gesamtwirtschaftlichen Effekte der arbeitsmarktpolitischen Förderung Bezug genommen. Die unterschiedlichen und teilweise gegensätzlichen Vorschläge zur Implementierung und Ausgestaltung zusätzlich zu schaffender Arbeitsplätze sowie teilweise in sich widersprüchliche Konzepte verweisen allerdings auf erheblichen weiteren Klärungsbedarf.
Die verbreitete Befürwortung längerfristiger oder gar dauerhafter Förderung für eine im Vergleich zum Problem relativ kleine Gruppe von „arbeitsmarktfernen“ Personen erfordert kritische Nachfragen: Soll mit der Prognose der aktuellen Nichtintegrierbarkeit eines bestimmten Personenkreises implizit die Botschaft transportiert werden, dass die Masse der Arbeitslosen in reguläre Beschäftigung integrierbar ist und deshalb vergleichbare Angebote der Beschäftigungsförderung nicht benötigt? Sollen die zusätzlichen Fördermöglichkeiten eingerichtet werden, weil man nicht daran glaubt, Fehlsteuerungen in der arbeitsmarktpolitischen Förderung („Creaming“) künftig überwinden und die gesamte Palette der differenzierten Förderinstrumente passgenau einsetzen zu können?

Fragen stellen sich auch in Bezug auf ein Herangehen, dass die Diagnose mittelfristig nicht gegebener Vermittelbarkeit letztlich an Merkmale der Person bindet, Beschäftigungsfähigkeit folglich als absolute und nicht als relationale Kategorie fasst. Es dürfte kaum strittig sein, dass die Wahrscheinlichkeit eines Übergangs in Beschäftigung auch von der Arbeitsmarktlage beeinflusst ist. Im Rahmen der Analyse von Stellenbesetzungsprozessen im Bereich sogenannter einfacher Dienstleistungen konnte gezeigt werden, dass ein grundlegendes Problem für die Beschäftigungsperspektiven gering Qualifizierter darin besteht, dass sie mit Personen konkurrieren, die eine abgeschlossene Berufsausbildung haben. Aus diesem Grund sei eine Verbesserung ihrer Arbeitsmarktchancen am ehesten erreichbar, wenn es gelänge, Beschäftigung auf allen Qualifikationsebenen zu steigern (Hieming et al. 2005, S. 239). Abgesehen davon sind auch für die Gruppen der Langzeitarbeitslosen, gesundheitlich Eingeschränkten und gering Qualifizierten immer wieder Integrationserfolge - mit oder ohne Maßnahmen - belegt. Für die Gruppe der derzeit Langzeitarbeitslosen dürfte es deshalb weniger um eine dauerhafte Aufbewahrung in separierten Beschäftigungsbereichen gehen als vielmehr darum, erstens ihre allgemeine Konkurrenzfähigkeit auf dem Arbeitsmarkt durch Qualifizierung zu erhöhen und zweitens Hilfen zur Vermeidung dauerhafter Ausgrenzung zu erhalten. Dies kann durch eine zeitlich befristete, aber immer wiederkehrende und sinnvoll aufeinander aufbauende Zuweisungsmöglichkeit in Quali- 
fizierungs- oder Beschäftigungsmaßnahmen erreicht werden. Dieses Ziel aufzugeben, käme einer arbeitsmarkt- und beschäftigungspolitischen Bankrotterklärung gleich.

Für die Arbeitsmarktpolitik stellt sich die Aufgabe, wirklich individuell zugeschnittene Angebote an alle Gruppen von Arbeitslosen zu richten, entsprechend den regionalen und lokalen Möglichkeiten auch nachfrageerhöhende Maßnahmen einzusetzen und dabei von Beginn an eine Anschlussfähigkeit der Projekte zu planen. Dies ist nicht trivial. Die vielfach zu Recht geäußerte Kritik an der Praxis von ABM und Zusatzjobs muss ernst genommen und im Sinne einer Verbesserung der Qualität der Maßnahmen produktiv gemacht werden. Dies setzt ein ehrliches Engagement aller beteiligten Akteure voraus. Notwendig ist die Bereitschaft, Probleme offen zu legen und auf eine Instrumentalisierung der Arbeitsmarktpolitik für andere Zwecke (z. B.
Machtausübung im Interesse der eigenen Klientel in lokalen Beiräten, kurzfristiger Aktionismus vor Wahlen u. ä.) zu verzichten. Eine abstrakte Kritik an den Instrumenten geht am Problem vorbei, denn sie verfügen über Potenziale, die sich nicht automatisch umsetzen, sondern vielmehr erst im Prozess ihrer Implementation erschlossen werden können und müssen. Umgekehrt kann eine unprofessionelle Nutzung der Instrumente allerdings auch mit negativen Wirkungen verbunden sein.

Für eine langfristig erfolgreiche arbeitsmarktpolitische Beschäftigungsförderung sind zwei Voraussetzungen unabdingbar: Erstens müssen sich Arbeitsbedingungen, Arbeitsorganisation und Entgelte in geförderter Beschäftigung an den betrieblich üblichen „Normalitätsbedingungen“ orientieren, wenn eine Verdrängung regulärer Beschäftigung vermieden und Übergänge auf ungeförderte Arbeitsplätze ermöglicht werden sollen. Zweitens kann die Arbeitsmarktpolitik zwar eine Art „Lokomotivfunktion" übernehmen und gesellschaftlich nützliche Arbeiten organisieren, wo ungedeckte Bedarfe bestehen. Sie kann sich aber nicht am eigenen Schopf aus dem Sumpf ziehen. Wenn sich die in geförderter Beschäftigung gestarteten Tätigkeiten als nützlich erwiesen haben und diese weiterhin nicht durch Wirtschaftsunternehmen angeboten werden, muss nach Wegen gesucht werden, sie in öffentlich (teil-)finanzierte reguläre Beschäftigung zu überführen. Dies ist aus ökonomischen und sozialen Gründen sinnvoll. Dafür sind allerdings politische Entscheidungen erforderlich, die ihrerseits eine breite gesellschaftliche Akzeptanz benötigen. Ob diese Akzeptanz hergestellt werden kann, dürfte - zwar nicht nur, aber auch - von den arbeitsmarktpolitischen Akteuren und der Qualität ihrer Arbeit abhängen. 
Alt, H. (2007): Deutschlands Ehrgeiz am Arbeitsmarkt, in: Frankfurter Rundschau, 5. Februar

Bartelheimer, P./Wagner, A. (2005): Machbarkeitsstudie Arbeitsmarktmonitor. Voruntersuchung für ein Projekt zur wissenschaftsgestützten Begleitung der Umsetzung der neuen Arbeitsmarktgesetze (Hartz I bis IV), Hans-Böckler-Stiftung, Arbeitspapier 102, Düsseldorf

Bernhard, S./Hohmeyer, K./Jozwiak, E. (2006): Zweiter Arbeitsmarkt. Im Westen noch nichts Neues, IAB-Kurzbericht 24 vom 14.12., Nürnberg Brandner, K. (2007): JobPerspektive. Arbeit für Langzeitarbeitslose ohne Chancen auf dem regulären Arbeitsmarkt, www.klausbrandner.de/db/ docs/doc_13816_2007329143944.pdf

Bundesagentur für Arbeit (BA) (2004): Kompendium Aktive Arbeitsmarktpolitik, Nürnberg

Bundesagentur für Arbeit, Statistik (2007): Ausgewählte arbeitsmarktpolitische Instrumente - lange Zeitreihen (2000-2006) Quelle: http://www.pub.arbeitsamt.de/hst/services/statistik/detail/f.html Bundesanstalt für Arbeit (BA) (1978): Überlegungen II zu einer vorausschauenden Arbeitsmarktpolitik, Nürnberg

Bundesministerium für Arbeit und Soziales (BMAS) (2006): Bericht 2006 zur Wirkung der Umsetzung der Vorschläge der Kommission Moderne Dienstleistungen am Arbeitsmarkt (ohne Grundsicherung für Arbeitsuchende), Berlin

Bundesministerium für Arbeit und Soziales (BMAS) (Hrsg.) (2007): Material zur Information: Bericht der "Arbeitsgruppe Arbeitsmarkt" , Berlin COMPASS/IMU/SÖSTRA/PIW/Universität Hamburg (2006): Evaluation der Maßnahmen zur Umsetzung der Vorschläge der Hartz-Kommission. Arbeitspaket 1: Wirksamkeit der Instrumente. Modul 1c: Arbeitsbeschaffungsmaßnahmen, Berlin, Bremen, Hamburg

Deutscher Bundestag, Ausschuss für Arbeit und Soziales (2007): Materialien zur öffentlichen Anhörung von Sachverständigen in Berlin am 7. Mai 2007 zum Antrag der Fraktion DIE LINKE. "Für eine Ausweitung und eine neue Qualität öffentlich finanzierter Beschäftigung“ und zum Antrag der Fraktion BÜNDNIS 90/DIE GRÜNEN "Arbeit statt Arbeitslosigkeit finanzieren“, Zusammenstellung der schriftlichen Stellungnahmen. Ausschussdrucksache 16(11)610, Berlin

Deutscher Gewerkschaftsbund (2006): Öffentlich geförderte Beschäftigung für Menschen mit geringen Arbeitsmarktchancen ausbauen, Arbeitsmarkt aktuell 5, Berlin

Fraktion BÜNDNIS 90/DIE GRÜNEN (2006): Arbeit statt Arbeitslosigkeit finanzieren, BT-Drs. 16/2652

Fraktion Die Linke (2006): Für eine Ausweitung und eine neue Qualität öffentlich finanzierter Beschäftigung, BT-Drs. 16/2504

Fuchs, L./Troost, J. (2003): Kommunale Beschäftigungsförderung. Ergebnisse einer Umfrage über Hilfen zur Arbeit nach BSHG und Arbeitsbeschaffungsmaßnahmen nach SGB III (im Auftrag des Deutschen Städtetages), Köln

Haberkorn, M. (2007): Wir brauchen keinen dritten Arbeitsmarkt für besonders Marktferne, sondern einen zweiten für besondere Marktnähe, Berlin. Download: http://www.bvaa-online.de/obj/DokumenteArbeitsmarkt/175keindritterAM
Hieming, B./Jaehrling,K./Kalina, T./Vanselow, A./Weinkopf, C. (2005): Stellenbesetzungsprozesse im Bereich "einfacher" Dienstleistungen, Berlin

Hohendanner, C. (2007): Verdrängen Ein-Euro-Jobs sozialversicherungspflichtige Beschäftigung in den Betrieben?, IAB Discussion-Paper 8, Nürnberg Hohmeyer, K./Schöll, C./Wolff, J. (2006): Arbeitsgelegenheiten in der Entgeltvariante. Viele Zielgruppen werden noch vernachlässigt, IAB-Forschungsbericht 22, Nürnberg

Kettner, A./Rebien, M. (2007): Soziale Arbeitsgelegenheiten. Einsatz und Wirkungsweise aus betrieblicher und arbeitsmarktpolitischer Perspektive, IAB-Forschungsbericht 2, Nürnberg

Knuth, M. (1996): Drehscheiben im Strukturwandel. Agenturen für Mobilitäts-, Arbeits- und Strukturförderung, Berlin

Knuth, M./Wagner, A. (1995): Arbeitsmarktpolitische Innovationen im ostdeutschen Transformationsprozess, in: Nolte, D./Sitte, R./Wagner, A. (Hrsg.): Wirtschaftliche und soziale Einheit Deutschlands. Eine Bilanz, Köln, S. 288-309

Koalitionsarbeitsgruppe (2003): Eckpunkte für ein Drittes und Viertes Gesetz für moderne Dienstleistungen am Arbeitsmarkt, Berlin, 26. Juni Koch, S./Kupka, P. (2007): Geförderte Beschäftigung für leistungsgeminderte Langzeitarbeitslose?, Friedrich-Ebert-Stiftung, WISO Diskurs Expertisen und Dokumentationen zur Wirtschafts- und Sozialpolitik, März 2007, Bonn

Koße, S./Luschei, F./Schmitz-Mandrela, U./Trube, A./Weiß, C. (2003): Neue Arbeitsplätze durch ABM? Exemplarische und quantitative Studien über arbeitsplatzgenerierende Effekte im Rahmen von öffentlich geförderter Beschäftigung, IAB-Werkstattbericht vom 22.10., Nürnberg Schomburg, J. (2007): Wege und Irrwege der öffentlich geförderten Beschäftigung. Download: http://www.lag-arbeit-hessen.de/fileadmin/ user upload/Wege_und_Irrwege_0507.pdf

Seifert, H./Wagner, A. (1992): Arbeitsmarktpolitik in den neuen Bundesländern am Scheideweg?, in: WSI-Mitteilungen 9, S. 620-627

Spindler, H. (2005): Über die Umfunktionierung und Instrumentalisierung der "Ein-Euro-Jobs", in: Forum sozial (Zeitschrift des DBSH, Deutscher Berufsverband für soziale Arbeit ) Heft 2, S. 11-13 und Heft 3, S. 13-15 Trube, A. (1997): Zur Theorie und Empirie des Zweiten Arbeitsmarkts. Exemplarische Erörterungen und praktische Versuche zur sozio-ökonomischen Bewertung lokaler Beschäftigungsförderung, Münster Wagner, A. (1995): Zweiter Arbeitsmarkt mit neuem Anspruch?, in: Seifert, H. (Hrsg.): Reform der Arbeitsmarktpolitik, Köln, S. 206-240 Wolff, J./Hohmeyer, K. (2006): Förderung von arbeitslosen Personen im Rechtskreis des SGB II durch Arbeitsgelegenheiten: Bislang wenig zielgruppenorientiert, IAB-Forschungsbericht 10, Nürnberg 